

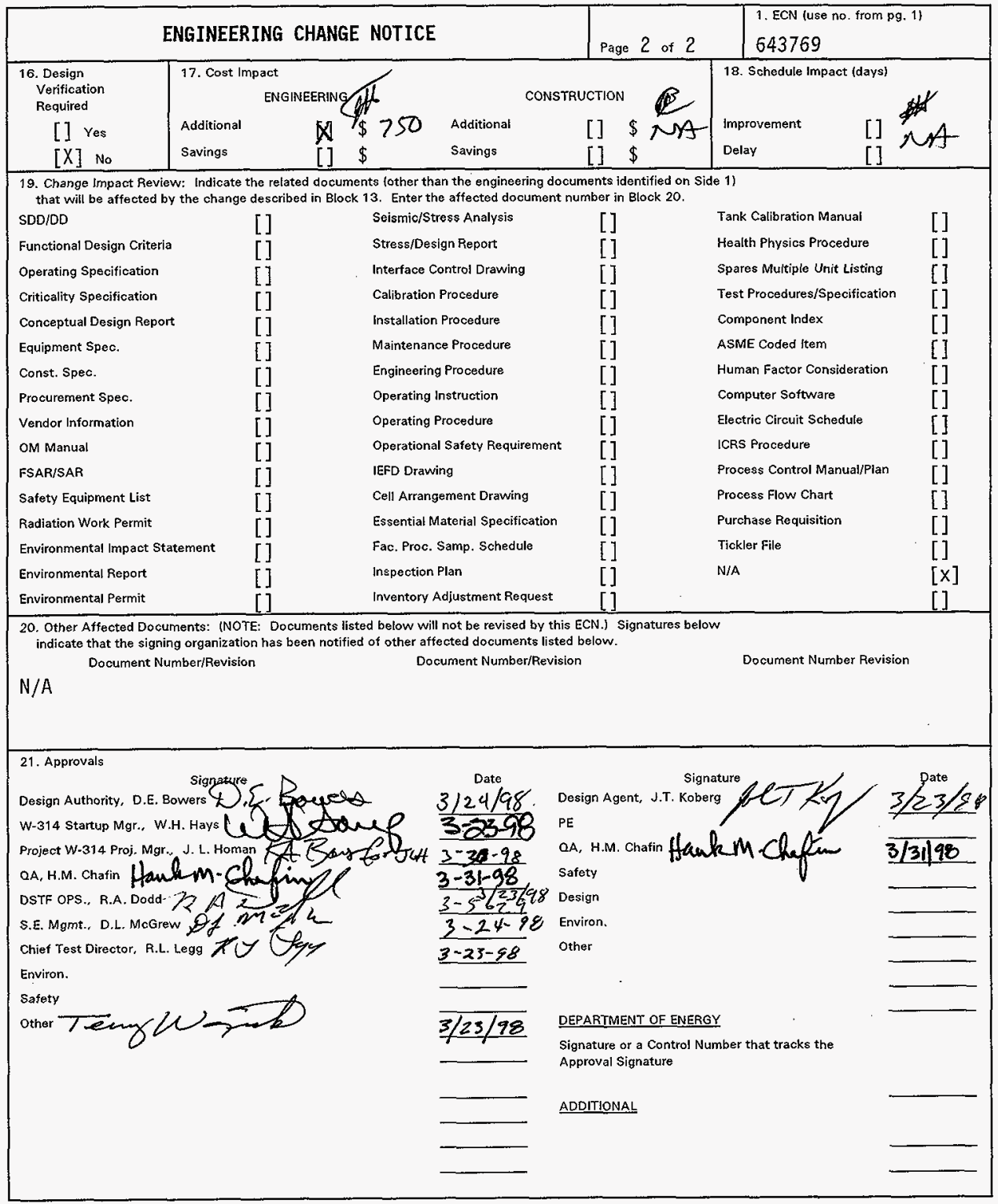


HNF-1902, Rev. 1

\title{
PROJECT W-314 SPECIFIC TEST AND EVALUATION PLAN 241-AN-B VALVE PIT
}

\author{
William H. Hays \\ Numatec Hanford Company, Richland, WA 99352 \\ U.S. Department of Energy Contract DE-AC06-96RL13200
}

\begin{tabular}{|c|c|c|}
\hline $\begin{array}{l}\text { EDT/ECN: } \\
\text { Org Code: } \\
\text { B\&R Code: }\end{array}$ & $\begin{array}{l}643769 \\
8 C 610 \\
\text { EW3130010 }\end{array}$ & $\begin{array}{l}\text { UC: } 2030 \\
\text { Charge Code: } \\
\text { Total Pages: }\end{array}$ \\
\hline
\end{tabular}

Key Words: Specific Test and Evaluation Plan, Project W-314, 241-AN-B Valve Pit

Abstract: The Specific Test and Evaluation Plan (STEP) defines the test and evaluation activities performed on the 241-AN-B Valve Pit for the W-314 Project.

TRADEMARK DISCLAIMER. Reference herein to any specific commercial product, process, or service by trade name, trademark, manufacturer, or otherwise, does not necessarily constitute or imply its endorsenent, recommendation, or fovoring by the United states Government or any agency thereof or its contractors or subcontractors.

Printed in the United States of Americe. To obtain copies of this document, contact: WHC/BCS Document Control Services, P.0. Box 1970, Mailstop H6-08, Richland H4 S9352, Phope (5092 372-2420: Fax (509) 376-4989.

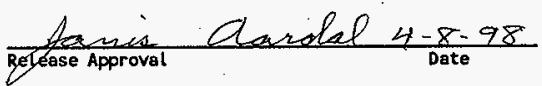

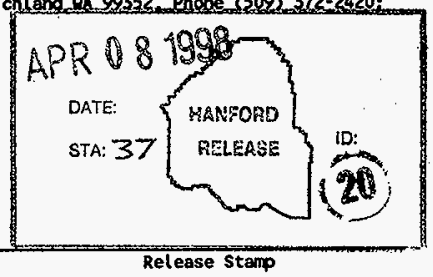


(2) Title

Specific Test and Evaluation Plan

CHANGE CONTROL RECORD

(3) Revision (4) Description of Change - Replace, Add, and Delete Pages

$0 \quad 17)$ EDT 622788 Dated December 30,1997

1 RS 241-AN-B Valve Pit Specific Test \&

Evaluation Plan, HNF-1902 Revise TabTe 5-1

by deleting 225A breaker replacement work

activity and correcting pane 7 numbers EDS-

DP-105 \& EDS-DP-106 to EDS-DP-103 \&

EDS-DP-105 respectively. Add drawing

reference for Construction Acceptance Test

1.4.C.B.C.15. Renumbered tests to parallel

required tests for each architectural unit.

Updated Logic Diagram. ECN 643769

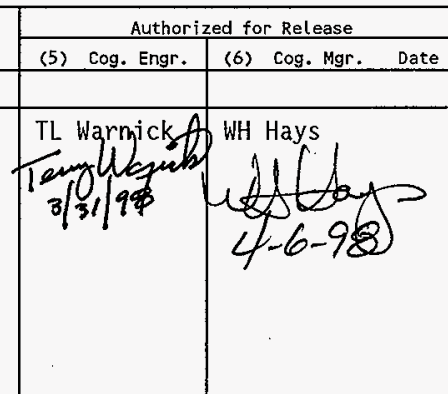


HNF-1902, REV. I

PROJECT W-314

SPECIFIC TEST AND EVALUATION PLAN

241-AN-B VALVE PIT 
TABLE OF CONTENTS

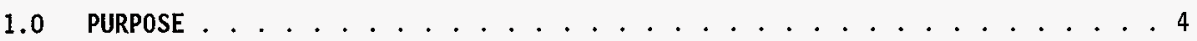

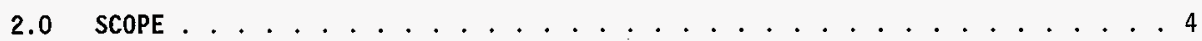

3.0 AdMINISTRATIVE . . . . . . . . . . . . . . . . . . . 4

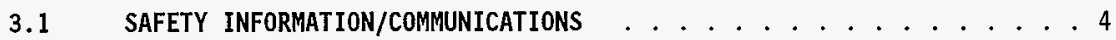

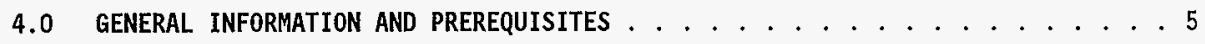

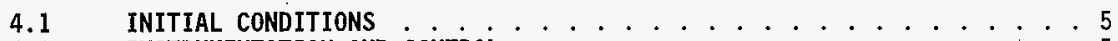

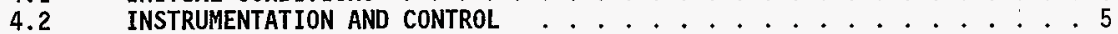

4.3 FUNCTIONAL TESTS ................... 5

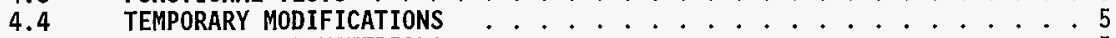

4.5 EQUIPMENT AND MATERIALS . . . . . . . . . . . . . 5

5.0 ASSIGNMENTS OF RESPONSIBILITY . . . . . . . . . . . . . . . . . . . 5

5.1 JOINT TEST REVIEW GROUP . . . . . . . . . . . . 6

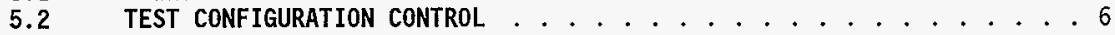

6.0 SYSTEM BOUNDARIES . . . . . . . . . . . . . . . . 6

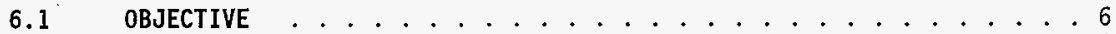

6.2 ADMINISTRATIVE CONTROLS .............. 6

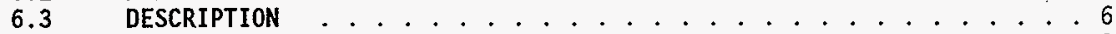

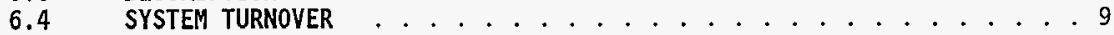

7.0 TEST AND ACCEPTANCE . . . . . . . . . . . . . . . . . . . 9

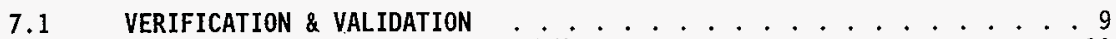

7.2 COMMERCIAL GRADE ITEM DEDICATION ................... 10

7.3 FACTORY ACCEPTANCE TESTING ................... 10

7.4 CONSTRUCTION ACCEPTANCE TESTING ........................ 11

7.5 ACCEPTANCE TESTING . . . . . . . . . . . . . . . 14

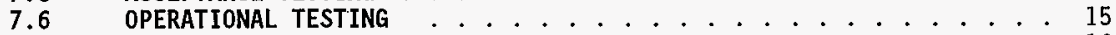

7.7 241-AN-B VALVE PIT TEST COMPLETION . . ............ 16

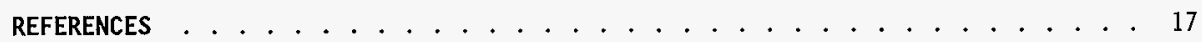

241-AN-B VALVE PIT TEST COMPLETION CHECKLIST . . . . . . . . . . . . . . 19 


\section{TABLE OF CONTENTS (CONT.)}

\section{LIST OF TABLES}

TABLE 5-1 241-AN-B VALVE PIT WORK AND TEST BOUNDARIES . . . . . . . . . 7

TABLE 7-2 COMMERCIAL GRADE ITEM DEDICATION ACTIVITIES . . . . . . . . . 10

TABLE $7-3$ FACTORY ACCEPTANCE TESTS . . . . . . . . . . . . . . . . . 10

TABLE $7-4 \quad$ CONSTRUCTION ACCEPTANCE TESTS . . . . . . . . . . . . . 11

TABLE 7-5 ACCEPTANCE TEST PROCEDURES . . . . . . . . . . . . . . . 14

TABLE 7-6 OPERATIONAL TEST PROCEDURES . . . . . . . . . . . . . 15

LIST OF FIGURES

FIGURE $1 \quad 241$-AN-B VALVE PIT LOGIC DIAGRAM . . . . . . . . . . . . 25 


\subsection{PURPOSE}

The purpose of this Specific Test and Evaluation PIan (STEP) is to provide a detailed written plan for the systematic testing of modifications made to the 241-AN-B Valve Pit by the $W-314$ Project. The STEP develops the outline for test procedures that verify the system's performance to the established Project design criteria. The STEP is a "lower tier" document based on the W-314 Test \& Evaluation Plan (TEP).

\section{$2.0 \quad$ SCOPE}

This STEP encompasses all testing activities required to demonstrate compliance to the project design criteria as it relates to the modifications of the 241-AN-B valve pit. The Project Design Specifications (PDS) identify the specific testing activities required for the Project. Testing includes Validations and Verifications (e.g., Commercial Grade Item Dedication activities...etc), Factory Acceptance Tests (FATs), installation tests and inspections, Construction Acceptance Tests (CATs), Acceptance Test Procedures (ATPs), Pre-0perational Test Procedures (POTPs), and Operational Test Procedures (OTPS). It should be noted that POTPS are not required for testing of the modifications to the 24l-AN-B Valve Pit. The STEP will be utilized in conjunction with the TEP for verification and validation.

\subsection{ADMINISTRATIVE}

\subsection{Safety Information/Communications}

Testing and inspection activities will be performed to the safety and communication procedures of the testing facility (e.g., shop, mockup, tank farm, vendor facility...etc.)

During the performance of 241-AN Tank Farm ATPS and OTPS, the Operations Test Director will be the direct 7 ine of communication with the Shift office. During abnormal and casualty situations, the Double-Shell Tank Farms Shift Manager will be the building emergency director/event commander. 


\subsection{GENERAL INFORMATION AND PREREOUISITES}

\subsection{Initial Conditions}

Prerequisite tests and al1 required construction, specific to the test, shall be completed prior to beginning any test. Test boundaries shall be verified as being appropriate and secure. Required locks and tags shall be correctly placed to allow performance of the test without disturbing the lock and tag. Required personnel shall be available and briefed on the steps of the test (a pre-job briefing is a prerequisite that must be satisfied before performing a Construction Work Package (CWP), ATP, and/or OTP). Required test apparatus sha11 be functional and set up in a safe configuration for the test. Installed and existing equipment, required for the test, shall be verified to be ready to operate in the test conditions.

\subsection{Measurement \& Test Equipment}

Instruments used will be specified per the test document and controlled per the performing agency's Measurement \& Test Equipment program.

\subsection{Functional Tests}

Functional tests, when required for calibration of equipment, will be performed prior to the associated ATP or OTP. Functional tests will be performed to approved documents.

\subsection{Temporary Modifications}

Temporary modifications may be required in 241-AN Tank Farm. Temporary modifications will be made per HNF-IP-0842, Vol. IV, section 4.5.

\subsection{Equipment and Materials}

Equipment installed by the project that fails during testing, or existing equipment that is damaged by testing, will be the responsibility of the construction contractor. Existing facility SSCs, not damaged but found defective, will be the responsibility of Doubie-She11 Tanks organization. Initiation of repairs will be accomp 7 ished by the creation of either a work package, using JCS, or a Construction Work Package (CWP).

\subsection{ASSIGNMENTS OF RESPONSIBILITY}

The design agent (FDNW) will provide test directors for the Acceptance Test Procedures. Tank Farm Operations will provide a qualified Tank Farm Shift Manager or Operations Engineer who will be the Test Directors for a 11 OTPs. This representative will accept the results of the ATPs for Tank Farm operations. Roles and responsibilities for construction. TWRS Operations, TFRSO Startup Group, W-314 Project Management, Acceptance Inspection, and Quality Assurance are defined in the W-314 TEP, Chapter three. 


\subsection{Joint Test Review Group}

The JTRG will consist of the Chief Test Director (from the TWRS Testing and Systems Readiness organization), Engineering manager (of the facility that will operate the equipment), W-314 Startup Manager, the Design Authority, applicable Test Director for each ATP \& OTP, and W-314 Project Manager. The JTRG provides Tank Farm Operations with a high level of confidence that the test can be performed safely and efficiently. The review by the JTRG provides a basis for test procedure approval and release. The JTRG conducts thorough reviews of ATPS and OTPs to ensure compliance with applicable procedural requirements, to ensure they can be performed safely, and to ensure the scope of testing and inspections provide a product that satisfies operational and safety requirements. The JTRG reviews test procedure data, and provides their recommendation concerning the final acceptance that test results satisfy the design specifications as stated in the ATPS, OTPS, or test plans.

\subsection{Test Configuration Control}

Testing shall be conducted using detailed test procedures and the 1 atest versions of all ECNs and Drawings for the Project. Configuration control shall conform to HNF-PRO-440 and HNF-PRO-226. Drawings shall be retained in project status by the W-314 Project until such time as al1 work on the 24l-AN-B valve pit is complete and accepted.

\subsection{SYSTEM BOUNDARIES}

\section{1 objective}

Specific system boundaries are identified for use in planning and implementing the various tests Tisted in this STEP. These system boundaries will provide safe work boundaries which allow testing to proceed in a safe environment, isolated from facility hazards.

\subsection{Administrative Controls}

Where actual physical isolations are not possible (e.g., pit nozzles...etc), Lock and Tag system (HNF-IP-0842, VoI. II, Sections 4.9.1 and 4.10.1), as a minimum, will be utilized to establish the required administrative controls.

\subsection{Description}

The table below lists the work and test boundaries for 241-AN-B valve pit work. These boundaries are estimates and may be modified at the time of execution to match current conditions in the farm and the job. This table will not be modifjed to reflect those changes. 
HNF-1902, REV. 1

TABLE 5-1 241-AN-B VALVE PIT WORK AND TEST BOUNDARIES

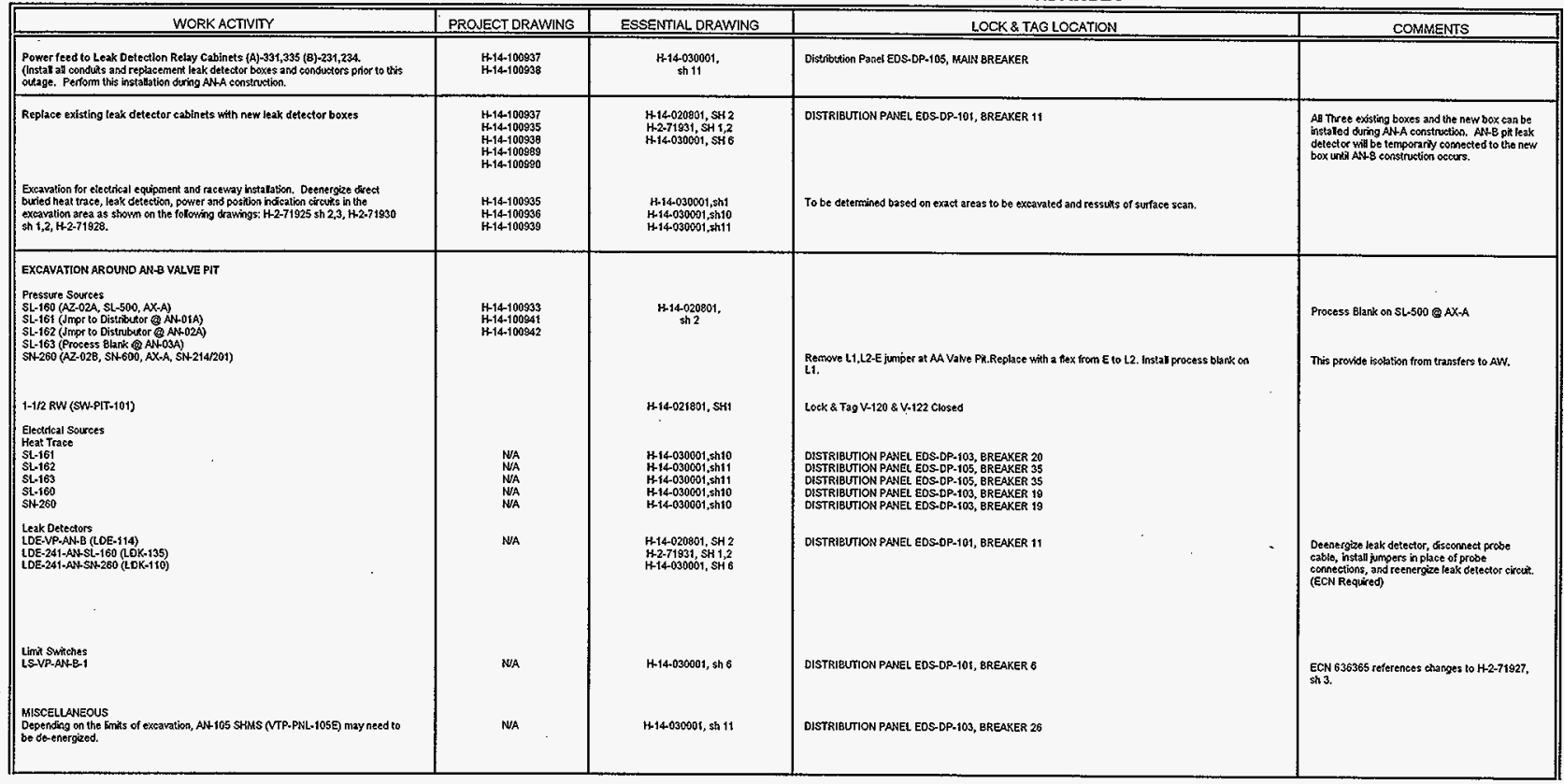




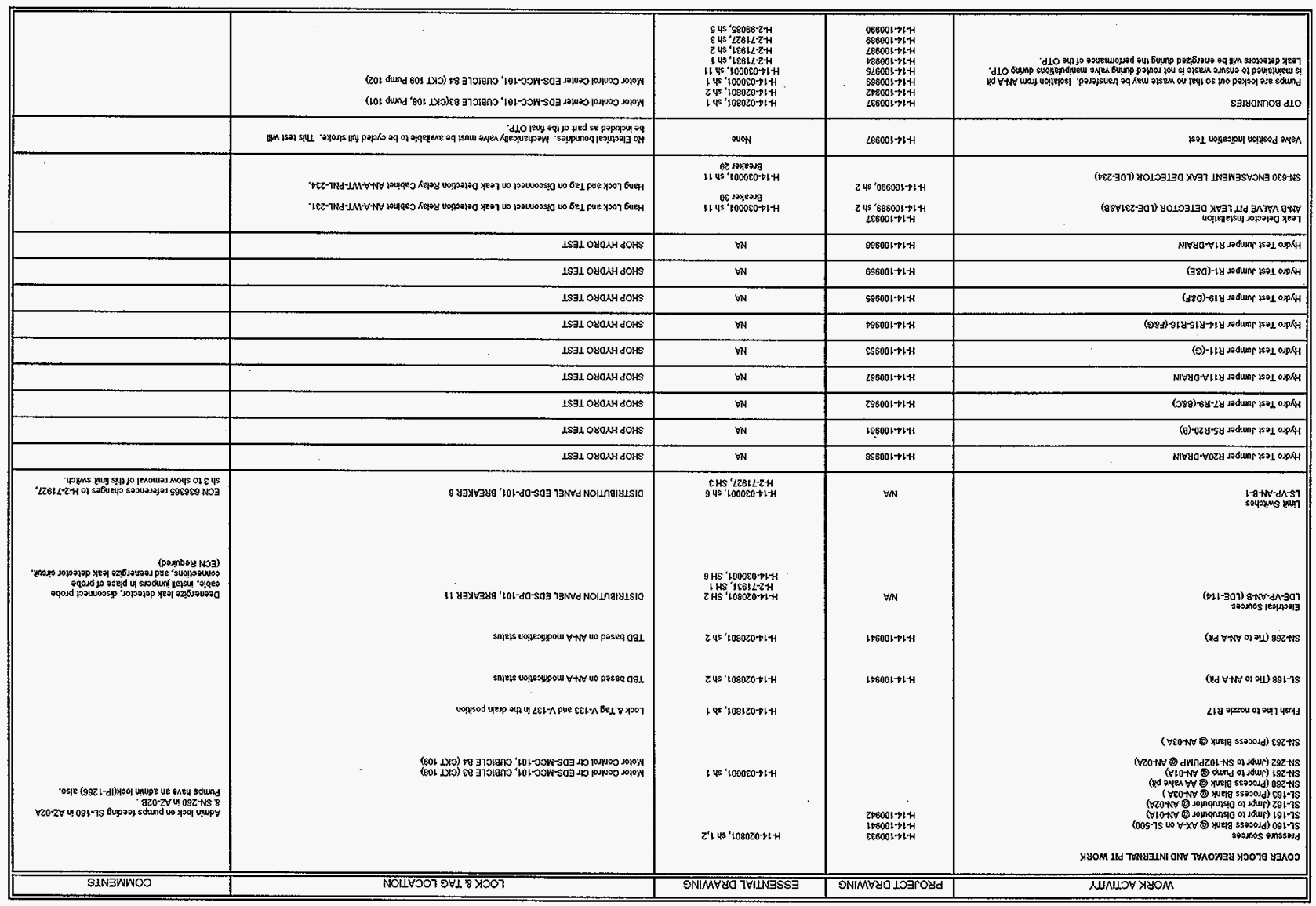

\section{I ‘ $\wedge$ Jy '206I-JNH}




\subsection{System Turnover}

Following completion and acceptance of all the required testing and testing data within a given boundary, the systems and equipment in that area will be ready for release to operations for their control and use. The SSC(s) will be released to TWRS Operations by using the Acceptance for Beneficial Use process described in the W-314 TEP (HNF-SD-W314-001).

\subsection{TEST AND ACCEPTANCE}

The Project $W-314$ 241-AN-B Valve Pit modification STEP includes design verifications performed by various methods including engineering analysis, Vendor data review, FATs and CATs performed in accordance with Procurement/Construction Specifications, drawings, and system functiona] tests performed in accordance with ATPs and OTPs. These verifications and tests will be documented in the W-3I4 Project 241-AN-B Requirements Verification Report (RVR), HNF-1534, Rev.0, and in specific test documentation (e.g., ATRs, OTRs, CWP...etc.). In addition, approved calibration and functional test procedures will be performed in preparation for the above listed tests.

\subsection{Validation and Verification}

A11 design requirements 1 isted in the Project Development Specifications are formally reviewed by analys is and examination for project compliance. Commercial grade equipment utilized in Safety Class applications, will be qualified per appropriate procedures and programs. Equipment used in non-safety class applications will be verified using vendor data to ensure the design requirements of the application are met. These reviews are documented in the 241-AN-B Valve Pit RVR. This report is listed in the reference section of this document. 


\subsection{Commercial Grade Item Dedication}

The following table lists the testing that will be performed to satisfy the Commercial Grade Item Dedication requirements for the modification of 241-AN-B Valve Pit. Note that acceptance criteria are included in the individual drawings, specifications, procedures, and CWPS.

TABLE 7-2

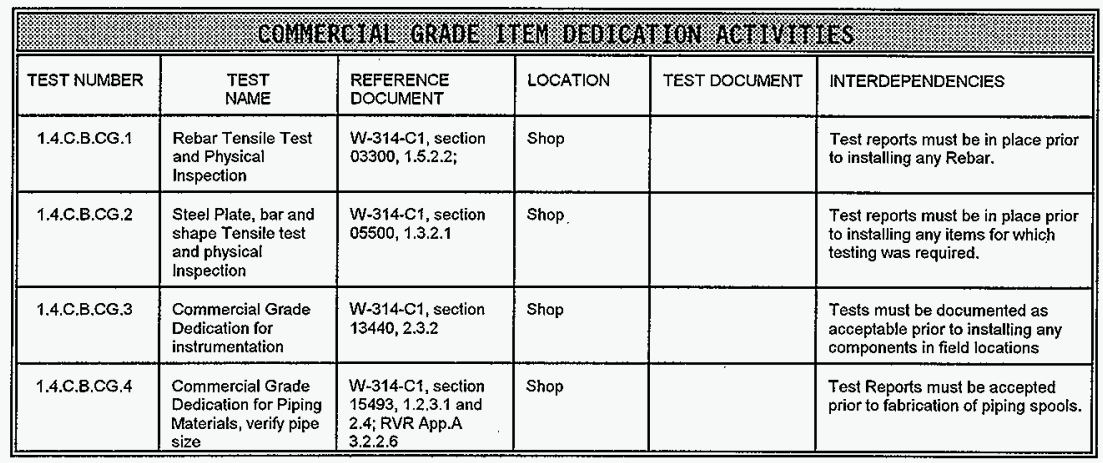

\subsection{Factory Acceptance Testing}

The following table lists the FATs that will be performed to modify 241-AN-B Valve Pit. Note that acceptance criteria are included in the individual drawings, specifications, procedures, and CWPs.

TABLE 7-3

\begin{tabular}{|c|c|c|c|c|c|}
\hline & & WOU & \% & 15. & \\
\hline TEST NUMBER & $\begin{array}{l}\text { TEST } \\
\text { NAME }\end{array}$ & $\begin{array}{l}\text { REFERENCE } \\
\text { DOCUMENT }\end{array}$ & LOCATION & TEST DOCUMENT & INTERDEPENDENCIES \\
\hline 1.4.C.B.F.1 & $\begin{array}{l}\text { Hydro and Leak } \\
\text { tests }\end{array}$ & $\begin{array}{l}\text { W-314-P1, section } \\
3.9\end{array}$ & Factory & & Prior to shipping \\
\hline
\end{tabular}




\subsection{Construction Acceptance Testing}

The table below lists the construction tests performed during fabrication, modification and installation to verify functionality of structures, systems and components following installation. AI inspection activities performed during construction are referenced in "ACCEPTANCE INSPECTION PLAN", W-314-C1-1, REV. B. Note that acceptance criteria are included in the individual test drawings, specifications, procedures, and CWPs.

TABLE 7-4

\begin{tabular}{|c|c|c|c|c|c|}
\hline 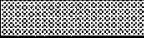 & & 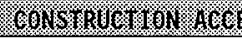 & $4 H_{10 \%}$ & S/ & \\
\hline TESTNUMBER & TEST & REFERENGE DOCQNENT & 10GATON & DOCEST & INTERDEPENDENCIES \\
\hline 1.4.C.A.C. 1 & In-Place Density Test & $\begin{array}{l}\text { W-314-C1, section } 02220 \text {, } \\
3.1,6.2\end{array}$ & Field & CWP & $\begin{array}{l}\text { Required prior to excavation for all } \\
\text { excavations }\end{array}$ \\
\hline 1.4.C.A.C.2 & $\begin{array}{l}\text { Backfill Density Test \& Cont. } \\
\text { Soil Use }\end{array}$ & $\begin{array}{l}\text { W-314-C1, section 02220, 3.3, } \\
\text { 3.4, 3.6.1; RVR App.A 3.3.1.6, } \\
\text { 3.3.1.7 }\end{array}$ & Field & CWP & During \& after backîll \\
\hline 1.4.C.A.C.3 & Concrete Slump Test & $\begin{array}{l}\text { W-314-C1, section 03300, } \\
\text { 1.5.2.1, 3.4.1; RVR App.A } \\
\text { 3.3.1.4, App.B 3.3.4.2 }\end{array}$ & Field & CWP & During concrete placement \\
\hline 1.4.C.A.C. 4 & Concrete Air Content Test & $\begin{array}{l}\text { W-314-C1, section } 03300 \\
\text { 1.5.2.1; RVR App.A } 3.3 .1 .4 \\
\text { App.B 3.3.4.2 }\end{array}$ & Field & CWP & During concrete placement \\
\hline 1.4.C.A.C.5 & Concrete Test Cyinders & $\begin{array}{l}\text { W-314-C1, section 03300, } \\
\text { 1.5.2.1; RVR App.A 3.3.1.4, } \\
\text { App.B 3.3.4.2 }\end{array}$ & Field, Lab & $\begin{array}{l}\text { Testing } \\
\text { Agency Report }\end{array}$ & $\begin{array}{l}\text { Cast cylinders during concrete } \\
\text { placement }\end{array}$ \\
\hline 1.4.C.A.C.6 & $\begin{array}{l}\text { Concrete Preplacement } \\
\text { Inspections }\end{array}$ & $\begin{array}{l}\text { W-314-C1, section 03300, 3.1, } \\
3.2 .1,3.2 .2 .1, \& 3.4 .2 ; R V R \\
\text { App.A 3.3.1.4; App.B 3.3.4.2, } \\
\text { 3.7.3.2.4 }\end{array}$ & Field & $\begin{array}{l}\text { CWP \& } \\
\text { Concrete pour } \\
\text { slip }\end{array}$ & Prior to Placing Concrete \\
\hline 1.4.C.A.C.7 & $\begin{array}{l}\text { Concrete Placement } \\
\text { Inspections }\end{array}$ & $\begin{array}{l}\text { W-314-CY, section 03300, } 3.2 \text { \& } \\
\text { 3.4.2, RVR App.A 3.3.1.4; } \\
\text { App.B 3.3.4.2 }\end{array}$ & Field & CWP & During concrete placement \\
\hline 1.4.C.A.C. 8 & $\begin{array}{l}\text { Concrete Post-Placement } \\
\text { Inspections }\end{array}$ & $\begin{array}{l}\text { W-314-C1, section } 03300,3.2 \text {, } \\
3,3 \& 3.4 .2\end{array}$ & Field & CWP & Prior to Cover block Installation. \\
\hline 1.4.C.A.C.9 & $\begin{array}{l}\text { Expansion Anchor } \\
\text { Inspections }\end{array}$ & W-314-C1, section $05055,3.4 .1$ & Field & CWP & During anchor installation \\
\hline 1.4.C.A.C.10 & $\begin{array}{l}\text { Structural Weld } \\
\text { Examinations }\end{array}$ & W-314-C1, section $05500,3.4 .1$ & Shop, Field & CWP & Prior to coating any structural pieces. \\
\hline 1.4.C.A.C.11 & $\begin{array}{l}\text { Cover Block Special } \\
\text { Protective Coating Surface } \\
\text { Preparation Inspection }\end{array}$ & $\begin{array}{l}\text { W-314-C1, section 09855, } 3.2 .2 \\
\text { \& 3.4.2; RVR App.D 3.3.1.2.1 }\end{array}$ & Shop. Field & CWP & Prior to application of coatings \\
\hline 1.4.C.A.C.12 & $\begin{array}{l}\text { Cover Block Special } \\
\text { Protective Coating } \\
\text { Application }\end{array}$ & $\begin{array}{l}\text { W-314-C1, section 09855, } \\
\text { 3.4.1; RVR App.D 3.2.2.1, } \\
3.2 .2 .2,3.2 .2 .8,3.2 .4,3.3 .1 .2 .2\end{array}$ & Shop, Field & CWP & During application of each coat \\
\hline 1.4.C.A.C.13 & $\begin{array}{l}\text { Pit Special Protective } \\
\text { Coating Surface Preparation } \\
\text { Inspection }\end{array}$ & $\begin{array}{l}\text { W-314-C1, section 09855, } 3.2 .2 \\
\text { \& 3.4.2; RVR App.D 3.3.1.2.1 }\end{array}$ & Shop, Field & CWP & Prior to application of coatings \\
\hline 1.4.C.A.C.14 & $\begin{array}{l}\text { Pit Special Protective } \\
\text { Coating Application }\end{array}$ & $\begin{array}{l}\text { W-314-C1, section 09855, } \\
3.4 .1 ; \text { RVR App.D 3.2.2.1, } \\
\text { 3.2.2.2, 3.2.2.8, 3.2.4, 3.3.1.2.2 }\end{array}$ & Shop, Field & CWP & During application of each coat \\
\hline 1.4.C.A.C.15 & $\begin{array}{l}\text { Cover Block Painting } \\
\text { Diagrams }\end{array}$ & $\begin{array}{l}\text { W-314-C1, section } 09855,3.3 .6 \\
\& \text { 3.4.2; RVR App.B 3.7.3.2.5 }\end{array}$ & Shop & CWP & $\begin{array}{l}\text { Verify painting diagram matches } \\
\text { information given in drawing s } \\
\text { referenced in section } 3.3 .6 \text {. }\end{array}$ \\
\hline
\end{tabular}




\begin{tabular}{|c|c|c|c|c|c|}
\hline & & 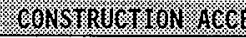 & 3rugl & & \\
\hline TEST NHMBER & ४ ग TEST & REFERENGE POCUMENT & LOATON & DOOESTENT & NTERDERENDENCIES \\
\hline 1.4.C.A.C.16 & $\begin{array}{l}\text { Leak Detector Fabrication } \\
\text { Wiring Continuity }\end{array}$ & $\begin{array}{l}\text { W-314-C1, section 13440, } \\
3.2 .1 \text { \& section } 16400,3.3 .1 .1\end{array}$ & Shop, Field & CWP & Complete prior to performing ATP. \\
\hline 1.4.C.A.C.17 & $\begin{array}{l}\text { Leak Detector Cabinet } \\
\text { Installation Wiring Continuity }\end{array}$ & $\begin{array}{l}\text { W-314-C1, section } 13440 \\
\text { 3.2.1.1 \& section }\{6400,3.3 .1 .1\end{array}$ & Shop, Field & CWP & Complete prior to performing ATP. \\
\hline 1.4.C.A.C. 18 & $\begin{array}{l}\text { Cover Block installation } \\
\text { Wiring Continuity }\end{array}$ & $\begin{array}{l}\text { W-314-C1, section 13440, } \\
\text { 3.2.1.1 \& section } 16400,3.3 .1 .1\end{array}$ & Shop, Field & $C W P$ & Complete prior to performing ATP. \\
\hline 1.4.C.A.C. 19 & $\begin{array}{l}\text { Terminal Box Conduit \& } \\
\text { Cable Installation Wiring } \\
\text { Continuity }\end{array}$ & $\begin{array}{l}\text { W-314-C1, section } 13440, \\
\text { 3.2.1.1 \& section } 16400,3.3 .1 .1\end{array}$ & Shop, Field & CWP & Complete prior to performing ATP. \\
\hline 1.4.C.A.C. 20 & $\begin{array}{l}\text { Valve Limit Switch Wiring } \\
\text { Continuity }\end{array}$ & $\begin{array}{l}\text { W-314-C1, section 13440, } \\
\text { 3.2.1.1 \& section } 16400,3.3 .1 .1\end{array}$ & Shop, Field & CWP & Complete prior to performing ATP. \\
\hline 1.4.C.A.C. 21 & $\begin{array}{l}\text { Leak Detector Inslallation } \\
\text { Wiring Continuity }\end{array}$ & $\begin{array}{l}\text { W-314-C1, section } 13440, \\
3.2 .1 .1 \text { \& section } 16400,3.3 .1 .1\end{array}$ & Shop, Field & CWP & Complete prior to performing ATP. \\
\hline 1.4.C.A.C.22 & $\begin{array}{l}\text { Cover Block Wiring Shielding } \\
\text { Resistance to Ground }\end{array}$ & $\begin{array}{l}\text { W-314-C1, section 13440, } \\
\text { 3.2.1.2. H-14-100936 SH3, } \\
\text { NOTE } 24\end{array}$ & Field & CWP & Complete prior to performing ATP \\
\hline 1.4.C.A.C.23 & $\begin{array}{l}\text { Terminal Box Conduit \& } \\
\text { Cable Shielding Resistance } \\
\text { to Ground }\end{array}$ & $\begin{array}{l}\text { W-314-C1, section 13440, } \\
3.2 .1 .2, \mathrm{H}-14-100936 \mathrm{SH} 3 \\
\text { NOTE } 24\end{array}$ & Field & CWP & Complete prior to performing ATP \\
\hline 1.4.C.A.C.24 & $\begin{array}{l}\text { Valve Limit Switch Shielding } \\
\text { Resistance to Ground }\end{array}$ & $\begin{array}{l}\text { W-314-C1, section 13440, } \\
\text { 3.2.1.2, H-14-100936.SH3, } \\
\text { NOTE } 24\end{array}$ & Field & CWP & Complete prior to performing ATP \\
\hline 1.4.C.A.C.25 & $\begin{array}{l}\text { Leak Detector Shlelding } \\
\text { Resistance to Ground }\end{array}$ & $\begin{array}{l}\text { W-314-C1, section } 13440, \\
3,2.1 .2, \mathrm{H}-14-100936 \mathrm{SH} 3 \text {, } \\
\text { NOTE } 24\end{array}$ & Field & CWP & Complete prior to performing ATP \\
\hline 1.4.C.A.C.26 & $\begin{array}{l}\text { Leak Dotector Fabrication } \\
\text { Voltage Verification }\end{array}$ & $\begin{array}{l}\text { W-314-C1, section } 16400 \text {, } \\
3.3 .1 .2\end{array}$ & Field & CWP & Prior to energizing equipment. \\
\hline 1.4.C.A.C. 27 & $\begin{array}{l}\text { Leak Detector Installation } \\
\text { Voltage Verification }\end{array}$ & $\begin{array}{l}\text { W-314-C1, section } 16400 \text {, } \\
3.3 .1 .2\end{array}$ & Field & CWP & Prior to energizing equipment. \\
\hline 1.4.C.A.C. 28 & $\begin{array}{l}\text { Holiday Testing of Piping } \\
\text { Protective Coatings }\end{array}$ & $\begin{array}{l}\text { W-314-C1, section } 15493 \text {, } \\
3.1 .6 .3\end{array}$ & Field & CWP & Prior to Backfill. \\
\hline 1.4.C.A.C.29 & $\begin{array}{l}\text { Piping Fabrication Welding } \\
\text { Non-Destructive Examination }\end{array}$ & $\begin{array}{l}\text { W-314-C1, section 15493, 3.2.1 } \\
\text { \& AppendiX A, 3.3 \& 4.1; RVR } \\
\text { App. A 3.2.2.4, 3.3.1.5, 3.3.4, } \\
\text { App. B 3.3.4.1 }\end{array}$ & Shop, Field & CWP & Prior to any form of pressure testing. \\
\hline 1.4.C.A.C.30 & $\begin{array}{l}\text { Jumper Fabrication Welding } \\
\text { Non-Destructive Examination }\end{array}$ & $\begin{array}{l}\text { W-314.C1, section } 15493,3.2 .1 \\
\text { \& Appendix A, 3.3 \& 4.1; RVR } \\
\text { App. A 3.3.1.5, 3.3.4, App. B } \\
\text { 3.3.4.1 }\end{array}$ & Shop, Field & CWP & Prior to any form of pressure testing. \\
\hline 1.4.C.A.C.31 & $\begin{array}{l}\text { Piping Fabrication } \\
\text { Cleanliness Inspection }\end{array}$ & $\begin{array}{l}\text { W-314-C1, section 15493, } \\
\text { 3.2.3.5; RVR App. A 3.3.4 }\end{array}$ & Shop Field & CWP & Prior to any form of pressure testing \\
\hline 1.4.C.A.C.32 & $\begin{array}{l}\text { Piping Fabrication Pressure } \\
\text { Tests }\end{array}$ & $\begin{array}{l}\text { W-314-C1, section 15493, } \\
\text { 3.2.4; RVR App. A 3.3.1.2 }\end{array}$ & Shop & CWP & $\begin{array}{l}\text { Test Nozzle spools prior to installation } \\
\text { in the field. }\end{array}$ \\
\hline 1.4.C.A.C.33 & $\begin{array}{l}\text { Piping Installation } \\
\text { Cleanliness Inspection }\end{array}$ & $\begin{array}{l}\text { W-314-C1, section } 15493 \text {, } \\
3.2 .3 .5 ; \text { RVR App. A } 3.3 .4\end{array}$ & Shop Field & CWP & Prior to any form of pressure testing \\
\hline 1.4.C.A.C.34 & $\begin{array}{l}\text { Piping Installation Pressure } \\
\text { Tests }\end{array}$ & $\begin{array}{l}\text { W-314-C1, section 15493, } \\
\text { 3.2.4; RVR App. A 3.3.1.2 }\end{array}$ & Shop & CWP & $\begin{array}{l}\text { Test Nozzle spools prior to installation } \\
\text { in the field. }\end{array}$ \\
\hline 1.4.C.A.C.35 & Jumper Pressure Tests & $\begin{array}{l}\text { W-314-C1, section } 15493 \text {, } \\
\text { Appendix A, } 3.6 \& 4.3\end{array}$ & Shop & CWP & $\begin{array}{l}\text { Test all Jumpers prior to installation in } \\
\text { the value pif }\end{array}$ \\
\hline 1.4.C.A.C.36 & $\begin{array}{l}\text { Jumper Fabrication } \\
\text { Inspections }\end{array}$ & $\begin{array}{l}\text { W-314-C1, section 15493, } \\
\text { Appendix A, } 4.2\end{array}$ & Shop & CWP & Prior to shop hydro \\
\hline
\end{tabular}




\begin{tabular}{|c|c|c|c|c|c|}
\hline 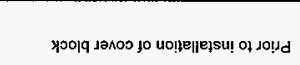 & SMO & plo! & 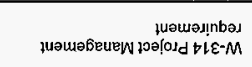 & 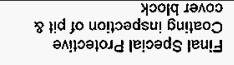 & 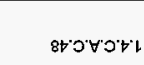 \\
\hline 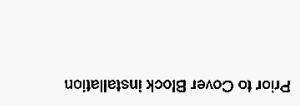 & AMO & pㅏㅏㅣㅋ. & 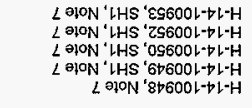 & 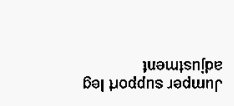 & $\angle D^{\circ} O \forall O^{\circ}$ \\
\hline 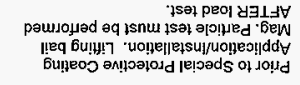 & dMO & dous & 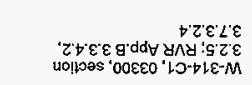 & 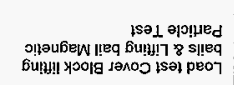 & 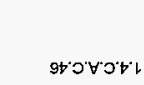 \\
\hline 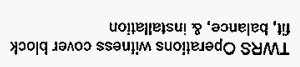 & dMO & PI리 & 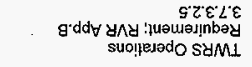 & 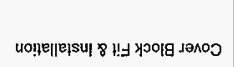 & St゚タ゚ロ \\
\hline $\begin{array}{r}\text { uope||ejsu! } \\
8 \text { әouejeq ssəuł! suonesədo słmL }\end{array}$ & $d M O$ & P[ध]!] & juawa!n bay suo!̣ejado SAML. & 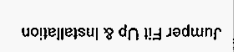 & 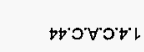 \\
\hline 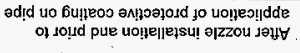 & dMO & p|ə!y & 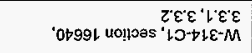 & 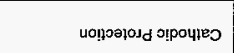 & ยナナัフォ゙ \\
\hline 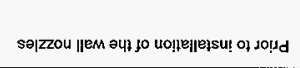 & $d M O$ & 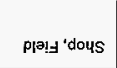 & 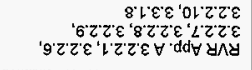 & 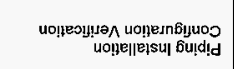 & 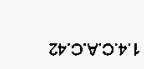 \\
\hline dio 6u!muoped of doldd & dMO & p|힙 & 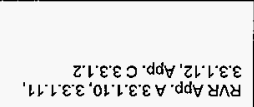 & 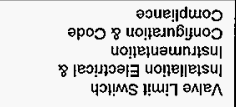 & 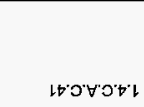 \\
\hline dLO Gujuuopəd ol solyd & dMO & Plø!̣ & 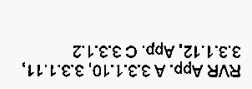 & 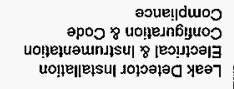 & 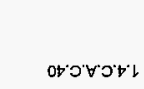 \\
\hline 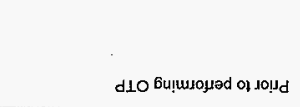 & $\triangle M O$ & plo!̣ & 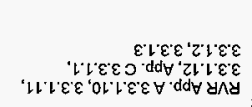 & 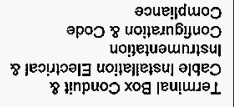 & 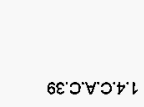 \\
\hline dLO Gulusoy, of do! dd & dMO & p)ə!y & 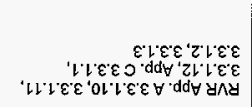 & 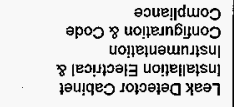 & 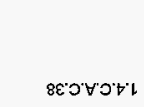 \\
\hline 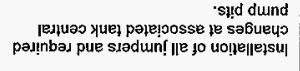 & 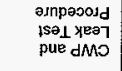 & ppoly & 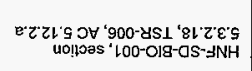 & 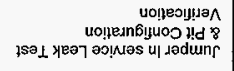 & $\angle E O \forall O \sigma^{\prime}$ \\
\hline 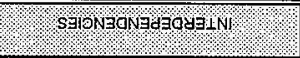 & HNBWOOOOH & NO11807 & 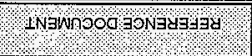 & 1531 & z 9 WNN $1 S A$ \\
\hline 85 & & 物 & 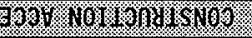 & & \\
\hline
\end{tabular}




\subsection{Acceptance Testing}

Acceptance testing will be conducted by utilizing ATPs. ATPs are separate "stand alone" documents which obtain their requirements from the applicable PDS, drawings, and vendor data. ATPs will ensure that the SSCS are fabricated and/or installed properly per the design. Detailed ATPs will be required after the CAT functional checks and/or inspections are completed and, will validate overall SSC installation as a whole. The acceptance criteria shall be specified in the test procedures and/or on approved drawings and specifications. These tests will be performed on components in their installed condition but may not exercise the entire system as an operational unit. The table below describes the ATPs that will be performed for 24l-AN-B Valve Pit modification and upgrade acceptance testing. An Acceptance Test Report (ATR) will be generated when a11 test exceptions have been cleared and the test has been successfully completed. Note that acceptance criteria are included in the individual test document.

TABLE 7-5

\begin{tabular}{|c|c|c|c|c|}
\hline \multicolumn{5}{|c|}{ AGubH } \\
\hline Q DOCUNENT & TEST DES SRIPTION & REFERENGE DOQUMENT & LOCATION & NTERDEPENCENGIES \\
\hline & Pit Leak Detection & $\begin{array}{l}\text { RVR App. A } 3.3 .2, \\
3.7 .2 .1 .2 .1,3.7 .2 .1 .2 .2, \\
3.7 .2 .1 .2 .3,3.7 .2 .1 .3 .1 \\
3.7 .2 .1 .3 .2,3.7 .2 .1 .3 .3 \\
\text { App. C } 3.2 .1 .1 .3,3.2 .2 .5\end{array}$ & Field & Leak Detector installed and energized \\
\hline & $\begin{array}{l}\text { Encasement Leak } \\
\text { Detection }\end{array}$ & $\begin{array}{l}\text { RVR App. A 3.2.4.5, 3.3.2, } \\
3.7 .2 .1 .2 .1,3.7 .2 .1 .2 .2 \\
3.7 .2 .1 .2 .3,3.7 .2 .1 .3 .1 \\
3.7 .2 .1 .3 .2,3.7 .2 .1 .3 .3\end{array}$ & Field & Leak Detector installed and energized \\
\hline & $\begin{array}{l}\text { Valve Manifold and } \\
\text { Indicators (Fosition } \\
\text { Switches) }\end{array}$ & $\begin{array}{l}\text { RVR App. B 3.3.2, } \\
\text { 3.7.2.1.1.4, 3.7.2.2.1, } \\
\text { 3.7.2.2.3, 3.7.2.2.4 }\end{array}$ & Field & $\begin{array}{l}\text { After equipment installation on cover } \\
\text { block }\end{array}$ \\
\hline
\end{tabular}




\subsection{Operational Testing}

Operational testing will be conducted by utilizing OTPS. OTPS will be $\mathrm{pl}$ lanned, scheduled, and conducted, on the installed SSCs, after completion and acceptance of all other test results. These detailed procedures shall determine if the installed SSCs meet the PDS functional requirements. Included in the OTP will be acceptance criteria to delineate pass/fail requirements. Operational testing is performed with actual plant equipment, operating procedures, and personne1. If an OTP has been generated but the testing of the corresponding SSC won't be performed for an extended period of time (e.g., a year or more), then the OTP must be reviewed again, prior to performance of the test, to ensure that it is still applicable. A Operational Test Report (OTR) will be generated when a11 test exceptions have been cleared and the test has been successfully completed. Testing of valve operators (with the valves connected), drain plug and leak detectors will be conducted with these components in their normal operating positions. Test conditions will meet the required code, design and authorization basis (i.e., Basis for Interim Operation, Technical Safety Requirements, and HNF-IP-1266, "TANK FARM OPERATIONS ADMINISTRATIVE CONTROLS") to verify that the system is functional and safe to operate. The following table describes the various sections of the OTP for 241-AN-B valve pit modifications. Note that acceptance criteria are included in the individual test document.

TABLE 7-6

\begin{tabular}{|c|c|c|c|c|c|}
\hline \multicolumn{6}{|c|}{ 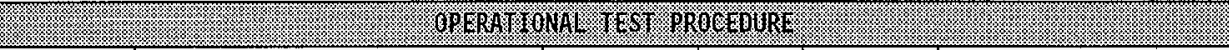 } \\
\hline Test Section & Testeescription & $\begin{array}{l}\text { Reference } \\
\text { Document }\end{array}$ & Lootation & Bocument & Interdependengles \\
\hline & $\begin{array}{l}\text { Architectural: } \\
\text { This section verifies that all components are } \\
\text { physically in place per the drawings, that they have } \\
\text { been correctly labeled, painted, etc. Items included } \\
\text { are electrical boxes, cover blocks, shlelding blocks, } \\
\text { valve operators, position indicators, yalve handles, } \\
\text { drain plug handles, and jumpers between cover } \\
\text { blocks and terminal boxes. Piping jumpers and } \\
\text { internal pit coating are speciffcally excluded from this } \\
\text { inspection as they will be verified as part of earlier } \\
\text { construction tests witnessed by operations. }\end{array}$ & 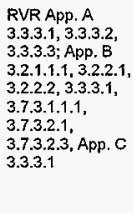 & Field & ОТР-314-002 & All equipment in place, \\
\hline & $\begin{array}{l}\text { Valve Operation: } \\
\text { This section tests the operation of the valve } \\
\text { operators and valve position indication. Operators } \\
\text { are verified to be able to move the valves and } \\
\text { remote indication of the position switches at the } \\
\text { terminal box is verified for all positions of each valve. } \\
\text { Verify functionality of valve locking devices. }\end{array}$ & & Field & OTP-314-002 & $\begin{array}{l}\text { All equipment in place. Locks and } \\
\text { tags in place to prevent inadvertent } \\
\text { transfer of waste. }\end{array}$ \\
\hline & $\begin{array}{l}\text { Leak Detector and Drain plug Operation: } \\
\text { This section verifies the operation of the leak } \\
\text { detector and the drain plug by adding water to the pit } \\
\text { until the leak detector actuates the alarms at } 271-A N \\
\text { and all other appropriate locations, and actuates the } \\
\text { master pump shutdown. Acceptance criteria for } \\
\text { operation is the amount and rate of water that the } \\
\text { system should detect per the design verification } \\
\text { report. This detection test shall be repeated no less } \\
\text { than three (3) times). }\end{array}$ & $\begin{array}{l}\text { RVR App. C } \\
3.2 .4 .4\end{array}$ & Field & OTP-314-002 & $\begin{array}{l}\text { All equipment in place, current } \\
\text { calibration of the leak detector and the } \\
\text { master pump shutdown system not in } \\
\text { alarm. }\end{array}$ \\
\hline
\end{tabular}


HNF-1902, REV. 1

\subsection{1-AN-B Valve Pit Test Completion}

241-AN-B Valve Pit testing will be considered successfully completed when the TEST COMPLETION CHECKLIST (attached to the end of this STEP) is completely signed off. This checklist is a "stand alone" document that will be 7 isted on the 241-AN-B Valve Pit ABU

(HNF-SD-W314-ABU-003) and included in the turnover of documents. This checklist will be completed by the W-314 Startup Manager or his designee. 


\section{REFERENCES}

HNF-PR0-229, Technical Procedure Standard, Fluor Daniel Hanford Company, Richland, Washington.

HNF-PR0-242, Rev. 1, Engineering Drawing Requirements, Fluor DanieT Hanford Company, Richland, Washington.

HNF-PR0-440, Engineering Document Change Control Requirements, Fluor Daniel Hanford Company, Richland, Washington.

HNF-PR0-446, Testing Practices Requirements, Fluor Daniel Hanford Company, Richland, Washington.

HNF-PR0-572, Project Acceptance and Closeout, Fluor Daniel Hanford Company, Richland, Washington.

W-314-C1, Construction Specification for Tank Farm Restoration and Safe operations AN Valve Pit Upgrades, Fluor Daniel Northwest, Inc., Richland, Washington.

W-314-P1, Procurement Specification Ba77 Valves AN Valve Pit Upgrades, Fluor Daniel Northwest, Inc., Richland, Washington.

W-314-P2, Procurement Specification Impact Wrench AN Valve Pit Upgrades, Fluor Daniel Northwest, Inc., Richland, Washington.

HNF-SD-W314-PDS-001, Rev. 0, Project Development Specification - Transfer Piping, Fluor Daniel Northwest, Inc., Richland, Washington.

HNF-SD-W314-PDS-002; Rev. 0, Project Development Specification for Valve Pit Manifold, Fluor Daniel Northwest, Inc., Richland, Washington.

HNF-SD-W314-PDS-003, Rev. 0, Project Development Specification for Pit Leak Detection, Fluor Daniel Northwest, Inc., Richland, Washington.

HNF-SD-W314-PDS-005, Rev. 0, Project Deve7opment Specification for Special Protective Coating, Fluor Daniel Northwest, Inc., Richland, Washington.

HNF-1534, Rev. 0, Requirements Verification Report for AN Valve Pit Upgrades (WBS 1.4.C), Fluor Daniel Northwest, Inc., Richland, Washington.

HNF-SD-W314-ABU-003, Rev. 0, AN-B VALVE PIT UPGRADE Acceptance For Beneficial Use, Rev. 0, Numatec Hanford Corporation, Richland, Washington.

HNF-SD-W314-TEP-001, Rev. 0, Test and Evaluation P7an for W-314 Tank Farm Restoration and Safe Operations, Numatec Hanford Corporation, Richland, Washington.

HNF-CM-3-5, Document Control and Records Management, Fluor Daniel Hanford Company, Richland, Washington.

HNF-IP-0842, TWRS Administration, Fluor Daniel Hanford Company, Richland, Washington. 
HNF-1902, REV. 1

HNF-IP-1266, Tank Farm Operations Administrative Controls, Fluor Daniel Hanford Company, Richland, Washington.

HNF-SD-WM-BI0-001, Rev. F, TWRS Basis for Interim Operation, Fluor Danie] Hanford Corporation, Richiand, Washington.

W-314-C1-1, Rev. B, Acceptance Inspection P7an, Fluor Daniel Hanford Corporation, Richland, Washington. 


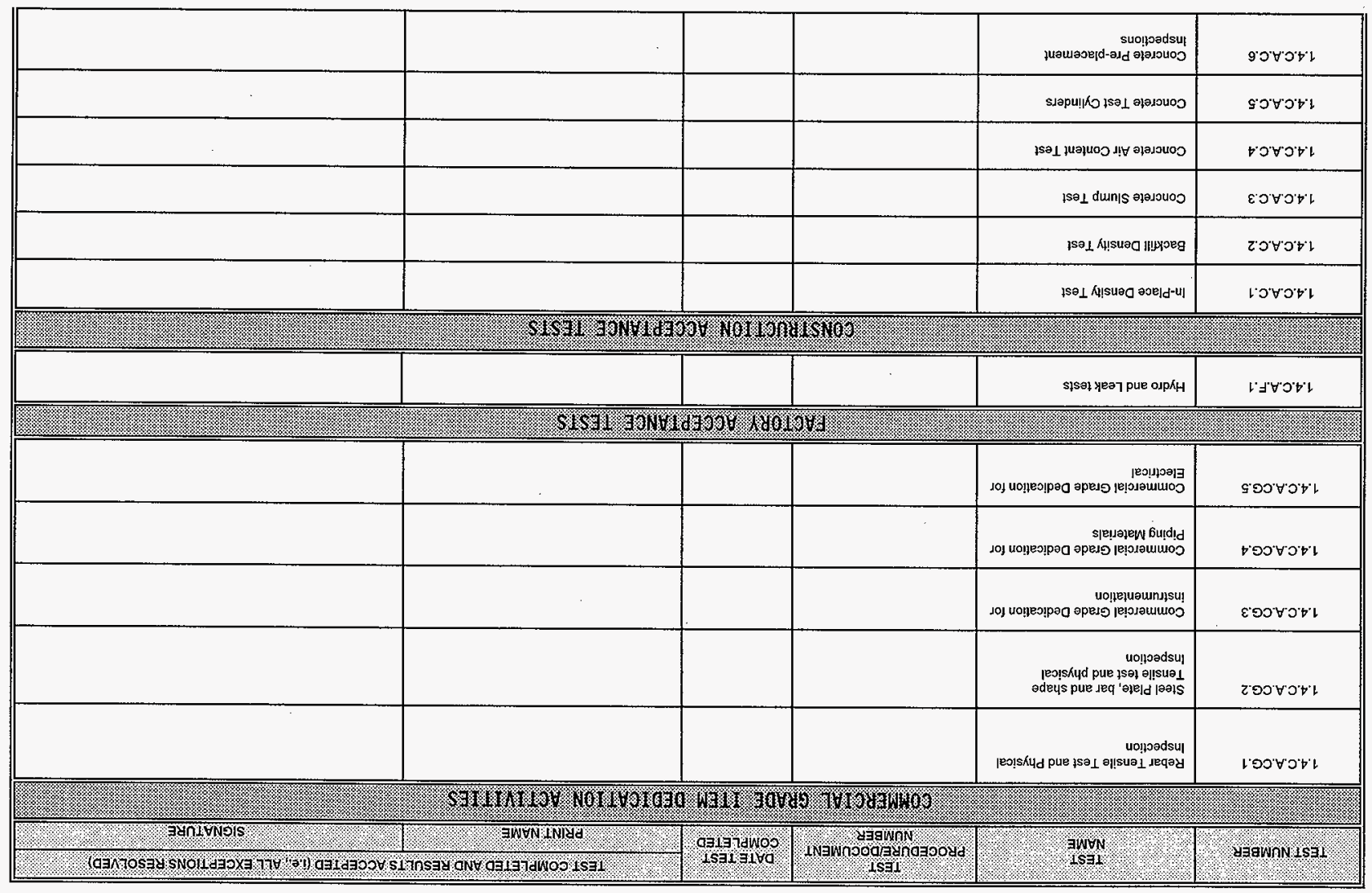

to $\exists$ Jyd

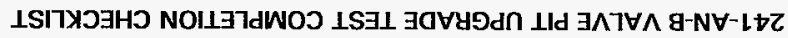

I ' $A \exists Y$ ' $206 I-J N H$ 
HNF-1902, REV. 1

241-AN-B VALVE PIT UPGRADE TEST COMPLETION CHECKLIST

PAGE OF

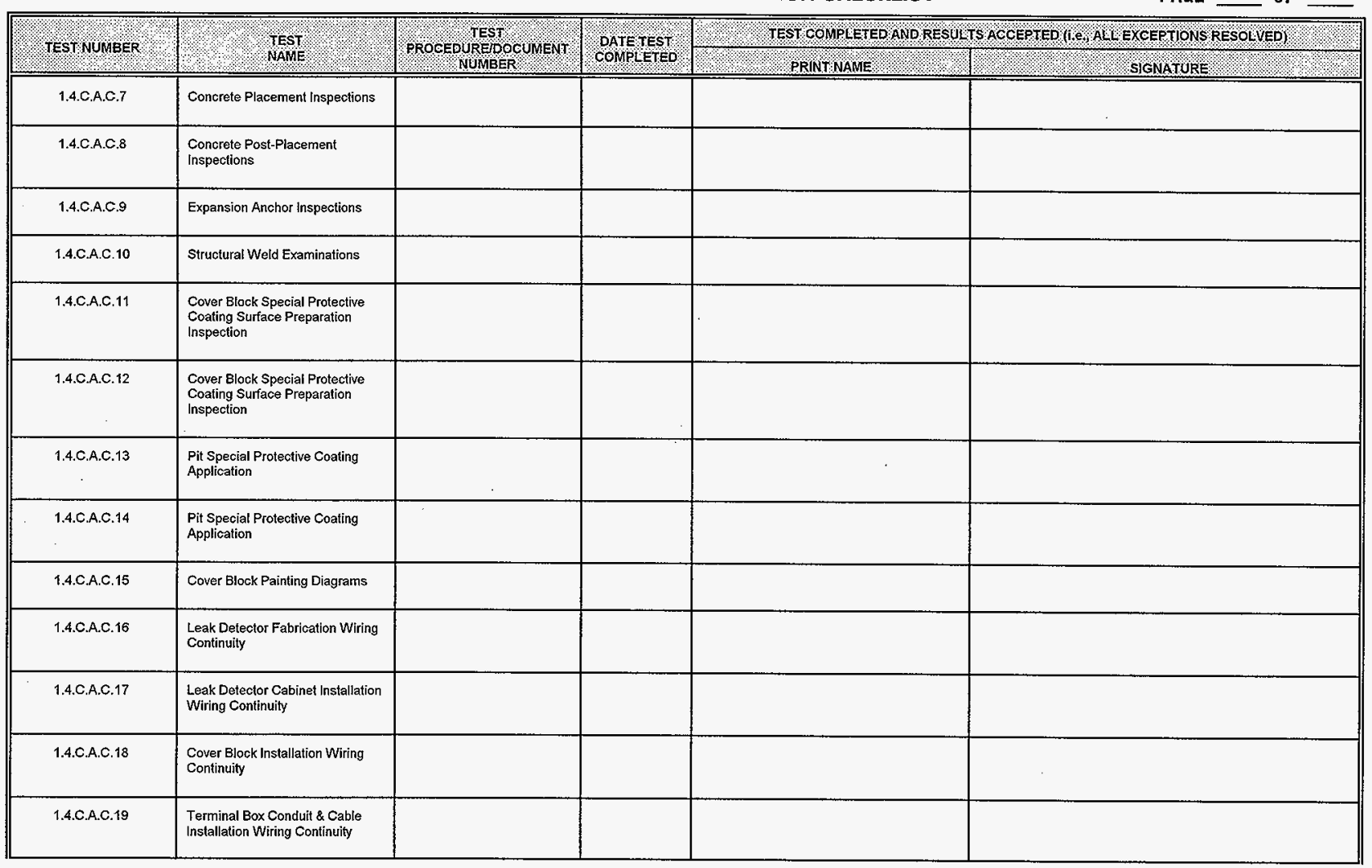




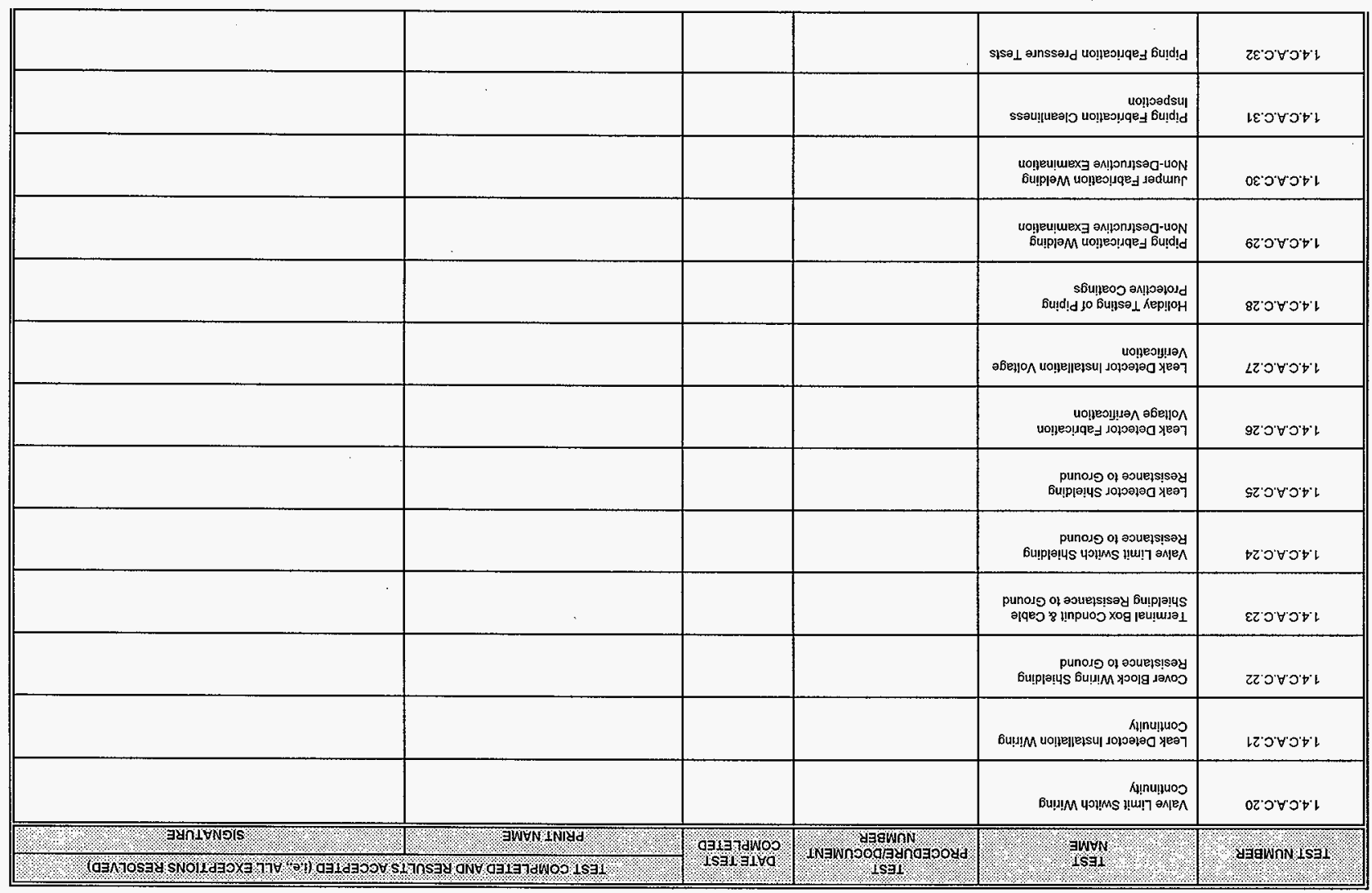




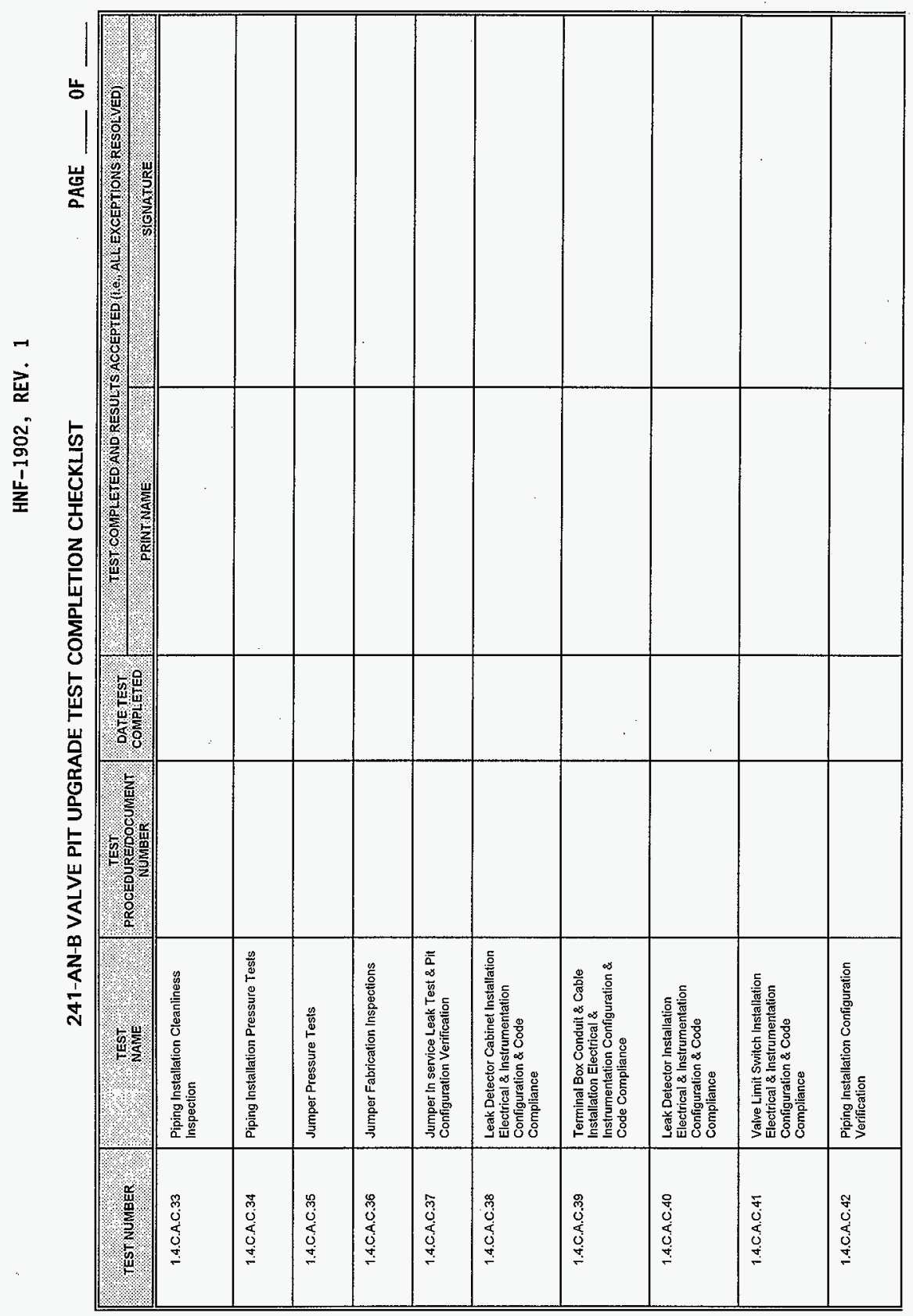

N 
HNF-1902, REV, 1

241-AN-B VALVE PIT UPGRADE TEST COMPLETION CHECKLIST

PAGE

OF

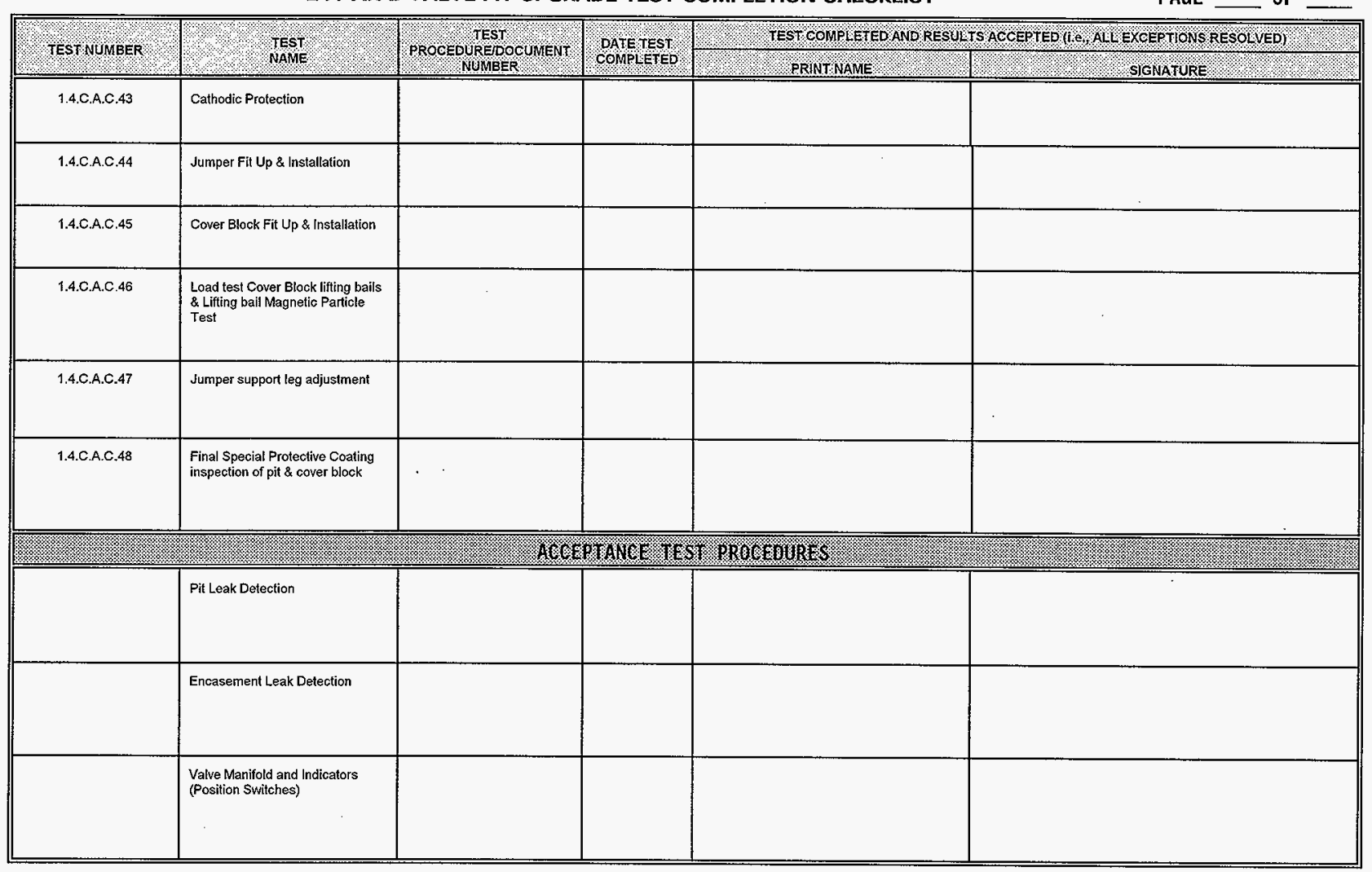




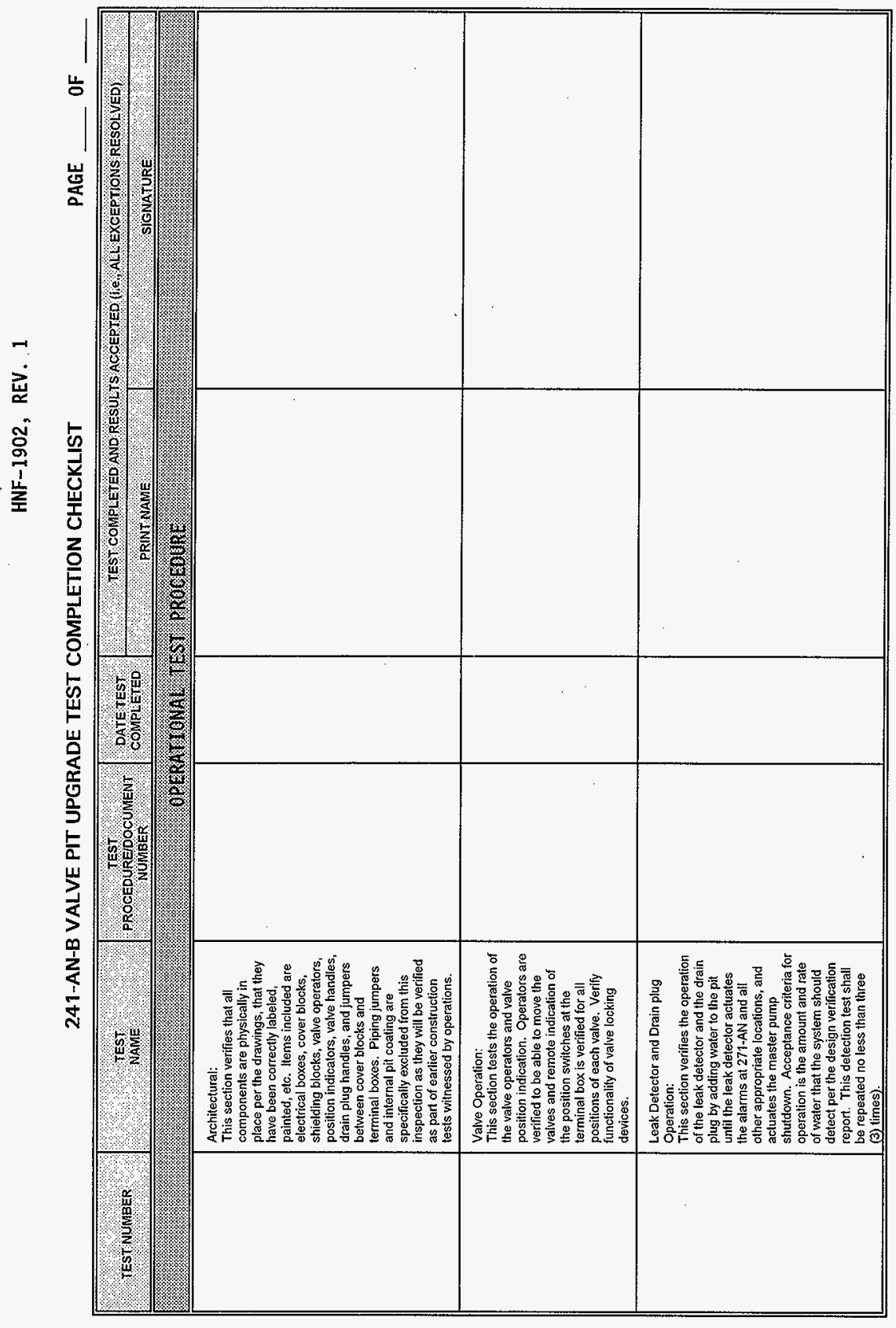

沗 


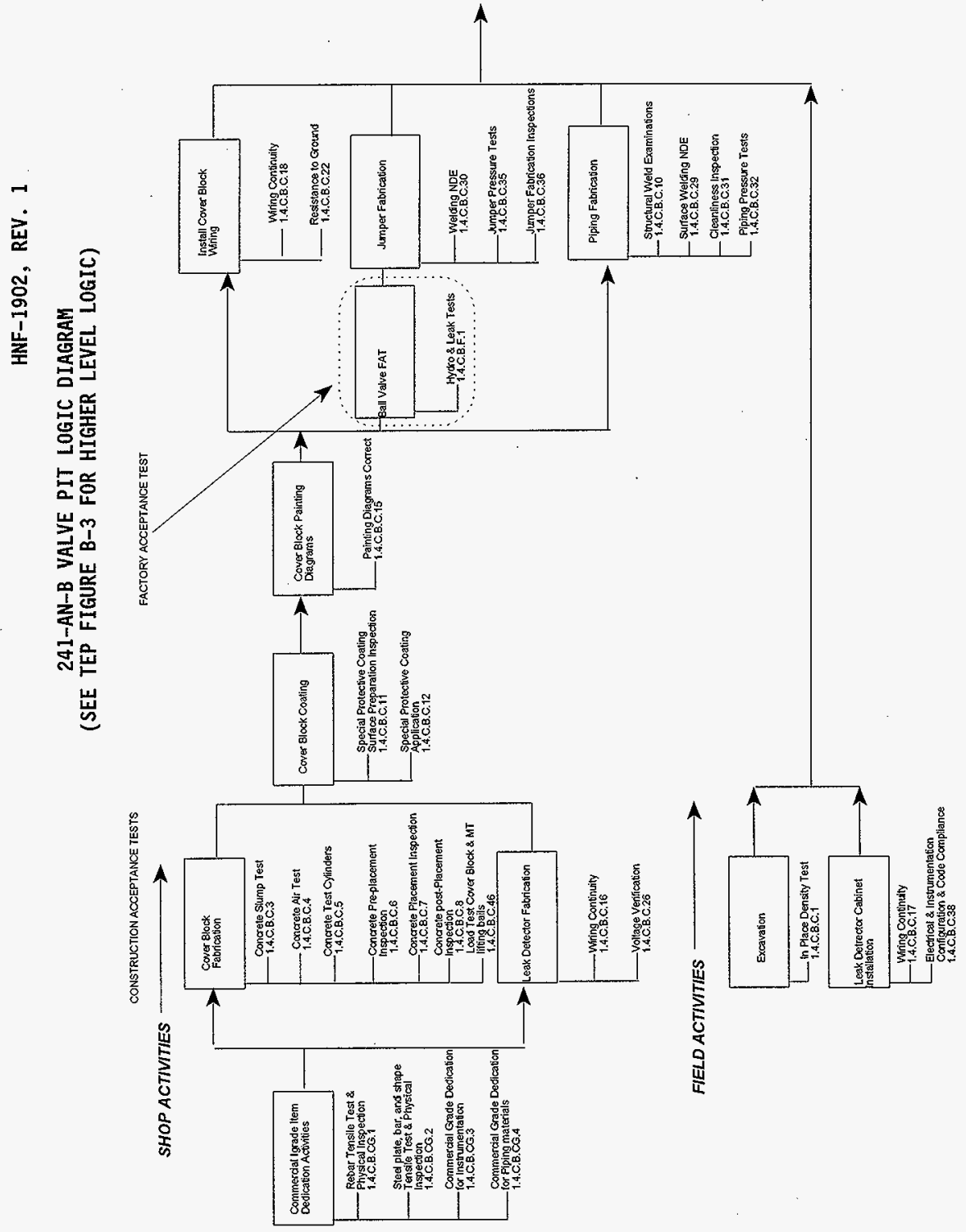


HNF-1902, REV. I

241-AN-B VALVE PIT LOGIC DIAGRAM [cont.]

(SEE TEP FIGURE B-3 FOR HIGHER LEVEL LOGIC)

CONSTRUCTION ACCEPTANCE TESTS (cont)

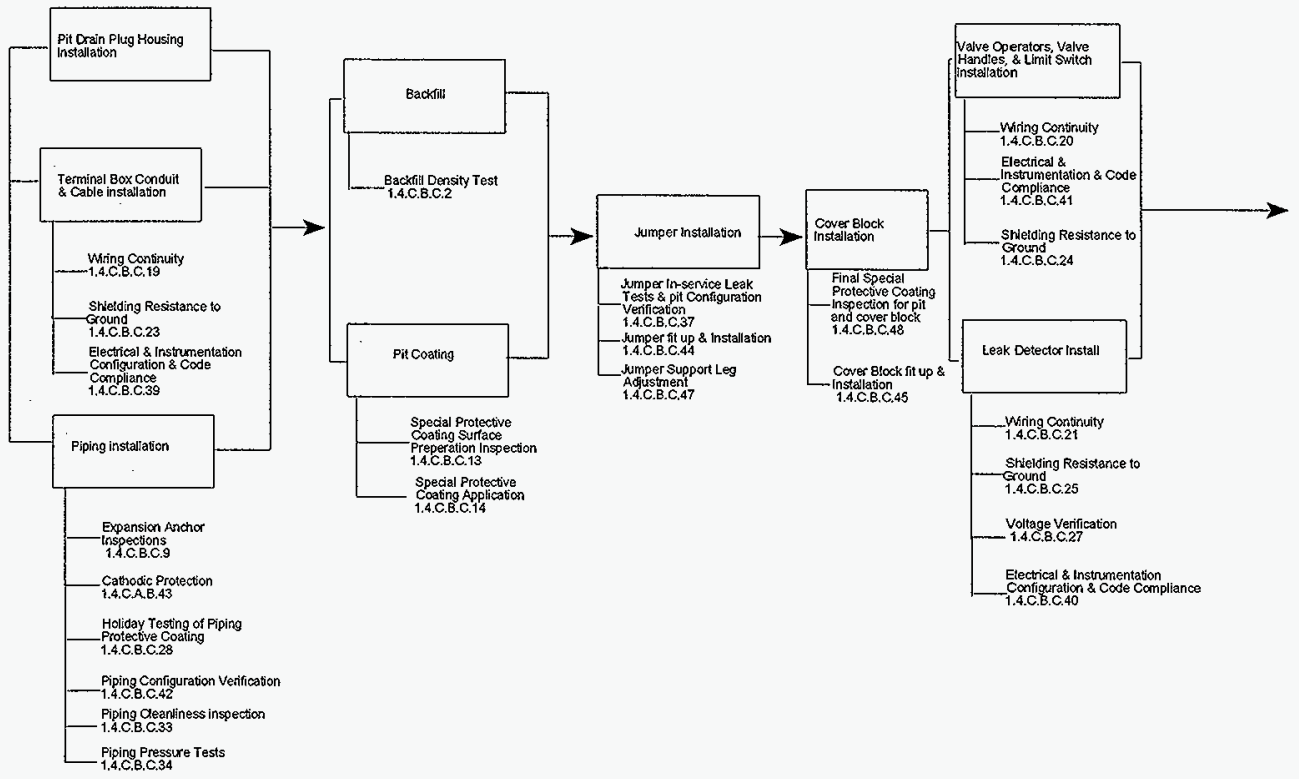



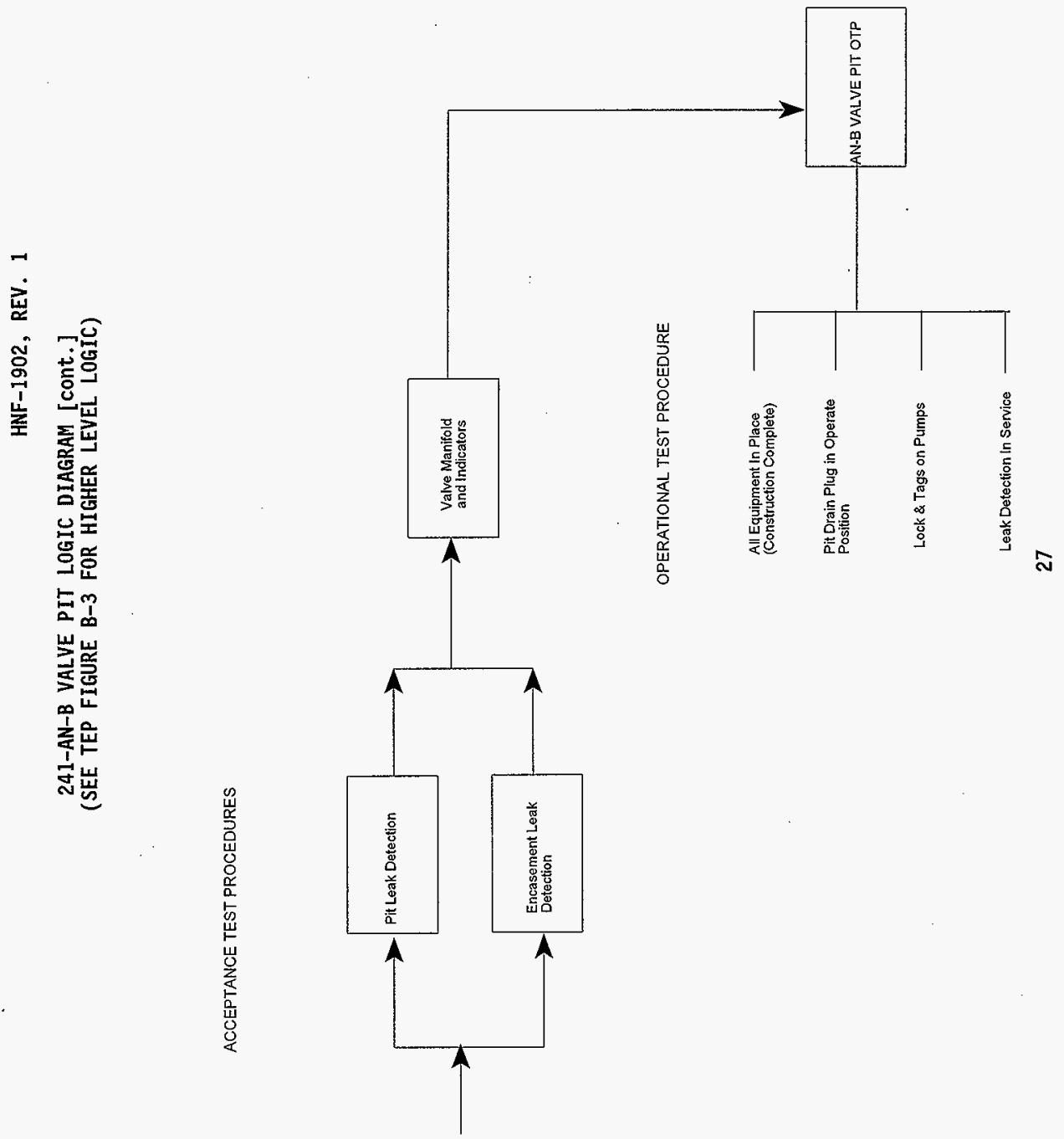


\section{DISTRIBUTION SHEET}

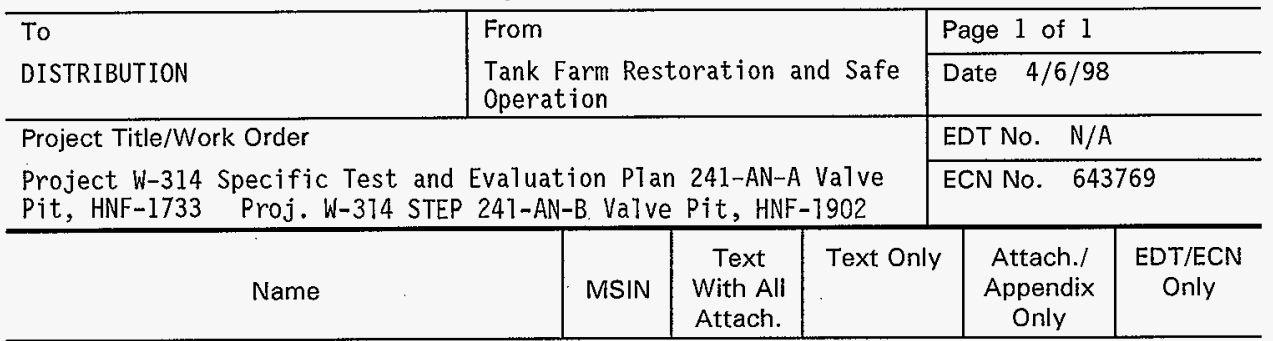

K. A. Boes

D. E. Bowers

R. L. Brown

H. M. Chafin

M. P. DeLozier

D. P. Devine

D. 0. Dobson

R. A. Dodd

J. D. Galbraith

P. A. Haine

C. E. Hatch

W. H. Hays

J. L. Homang

R. W. Jacobson

J. T. Koberg

S. K. Kujak

J. R. LaPointe

S. L. Leckband

R. L. Legg

D. L. McGrew

P. C. Miller

D. R. Nunamaker

E. A. Pacquet

T. K. Ravencraft

D. W. Reberger

M. D. Rickenbach

C. A. Rieck

B. L. Syverson

W. T. Thompson

M. W. Tiffany

T. L. Warnick

S. U. Zaman

Project Files

IPF \#23

Central Files
R3-25

S5-13

R3-38

R3-25

R2-50

S5-50

R2-50

S5-07

H5-49

R3-47

S4-49

E6-08

R2-58

R1-09

G3-12

B4-57

R2-88

R3-47

R2-50

R3-25

R1-51

T4-07

R3-47

S5-04

S5-13

G3-12

S2-48

G3-12

G3-21

R1-49

E6-11

R3-01

$\mathrm{R} 1-29$

T3-12

B] -07 $x$

$x$

$x$

$x$

$X$

$x$

$x$

$x$

$x$

$x$

$x$
$x$
$x$

$x$

$x$

$x$

$x$

$x$

$x$

$\mathrm{X}$

$X$

$x$

$x$

$x$

$x$

$x$

$x$

$x$

$x$
$x$
$x$

$x$
$x$

$x$

$x$

$x$

$x$

$\mathrm{x}$

$x$

$x$ (2) 